\title{
Selenium status is positively correlated with fish consumption in a cohort of high fish-eating pregnant women
}

\author{
M. S. Mulhern ${ }^{1}$, A. J. Yeates ${ }^{1}$, E. M. McSorley ${ }^{1}$, G. E. Watson ${ }^{2}$, G. J. Myers ${ }^{2}$, C. Shamlaye ${ }^{3}$, \\ J. Henderson ${ }^{3}$, E. van Wijngaarden ${ }^{2}$, P. W. Davidson ${ }^{2}$ and J. J. Strain ${ }^{1}$ \\ ${ }^{1}$ Northern Ireland Centre for Food and Health, University of Ulster, Coleraine, BT52 1SA, ${ }^{2}$ University of Rochester, \\ School of Medicine and Dentistry, NY, USA and ${ }^{3}$ Ministry of Health, Victoria, Mahé, Republic of Seychelles
}

It is widely accepted that diet during pregnancy plays a critical role in the health of the mother and the developing child. In particular, prenatal consumption of fish is known to be beneficial for child neurodevelopment and cognitive function ${ }^{(1)}$. Fish is the major dietary source of the long chain polyunsaturated fatty acids (LCPUFA), particularly the n-3 LCPUFA docosahexaenoic acid (DHA) and selenium. Whilst prenatal selenium status has been reported in some studies ${ }^{(2)}$, the correlation with fish consumption in a high-fish consuming pregnant population has not been investigated. Therefore, the aim of this study was to examine associations among selenium status, DHA concentrations and fish consumption during pregnancy in a cohort of high fish consumers.

A total of $n=1536$ pregnant women were recruited onto the Seychelles Child Development and Nutrition Study Cohort 2 and blood samples were collected at 28 weeks gestation. Serum selenium concentrations were quantified using inductively coupled plasma mass spectrometry in collision cell technology mode (XSERIES 1 X0339 ICP-MS, Thermo Scientific, US) and serum LCPUFA methyl esters were quantified using GC-MS, following lipid extraction, as previously described ${ }^{(3)}$. Fish consumption during pregnancy was self-reported using a specifically designed Food Use Questionnaire. Spearman correlation analyses were used to test associations between maternal selenium status, DHA concentrations and reported fish consumption.

The mean (SD) age of pregnant women at enrolment to the study was 26 (6) years and they reported consuming a mean (SD) of 8.5 (4.6) fish meals/week during pregnancy. Median $\left(5^{\text {th }}, 95^{\text {th }}\right.$ percentile) serum selenium status was $102.0(77.6,137.2) \mu \mathrm{g} / \mathrm{L}$ and $\mathrm{DHA}$ status was $0.180(0.062,0.330) \mathrm{mg} / \mathrm{ml}$. Reported fish consumption was significantly positively correlated with selenium concentrations $(\mathrm{r}=0.103, \mathrm{P}<0.0001)$ and DHA concentrations $(\mathrm{r}=0.054, \mathrm{P}=0.044)$. There was a significant positive correlation between selenium and DHA concentrations $(\mathrm{r}=0.132, \mathrm{P}<0.0001)$.

In this high fish-eating population, selenium status at 28 weeks gestation was within the normal reference range for healthy pregnant women in the third trimester ${ }^{(4)}$ and similar to that reported in other studies ${ }^{(2)}$. Fish consumption was a predictor of selenium status and DHA concentrations in this cohort. Further research is needed to investigate the effects of prenatal selenium status on pregnancy outcomes in a high-fish eating population.

This work was supported by grants from the US National Institute of Environmental Health Sciences, the National Institutes of Health and the Government of Seychelles.

1. Hibbeln JR, Davis JM, Steer C et al. (2007) Lancet 369(9561), 578-585.

2. Horton DK, Adetona O, Aguilar-Villalobos M et al. (2013) Nutr J 12, 80-90.

3. Bonham MP, Duffy EM, Wallace JMW et al. (2008) Prostag Leukotr Ess 78, 343-350.

4. Abbassi-Ghanavati M, Greer LG, Cunningham FG (2009) Obstet Gynecol 114(6), 1326-1331. 